\title{
YBC: a stellar bolometric corrections database with variable extinction coefficients
} Application to PARSEC isochrones ${ }^{\star}$

\author{
Yang Chen (陈洋) ${ }^{1}$, Léo Girardi ${ }^{2}$, Xiaoting Fu (符晓婷) ${ }^{3}$, Alessandro Bressan ${ }^{4}$, Bernhard Aringer ${ }^{1}$, Piero Dal Tio ${ }^{1,2}$, \\ Giada Pastorelli ${ }^{1}$, Paola Marigo ${ }^{1}$, Guglielmo Costa $^{4}$, and Xing Zhang (张星) ${ }^{5}$ \\ 1 Dipartimento di Fisica e Astronomia Galileo Galilei, Università di Padova, Vicolo dell'Osservatorio 3, 35122 Padova, Italy \\ e-mail: yang.chen@unipd.it \\ 2 Osservatorio Astronomico di Padova - INAF, Vicolo dell'Osservatorio 5, 35122 Padova, Italy \\ 3 The Kavli Institute for Astronomy and Astrophysics at Peking University, Beijing, PR China \\ e-mail: xiaoting. fu@pku.edu.cn \\ 4 SISSA, Via Bonomea 365, 34136 Trieste, Italy \\ 5 Department of Astronomy, University of Science and Technology of China, Chinese Academy of Sciences, Hefei, Anhui 230026, \\ PR China
}

Received 1 September 2019 / Accepted 17 October 2019

\begin{abstract}
We present the YBC database of stellar bolometric corrections, in which we homogenise widely used theoretical stellar spectral libraries and provide BCs for many popular photometric systems, including Gaia filters. The database can easily be extended to additional photometric systems and stellar spectral libraries. The web interface allows users to transform their catalogue of theoretical stellar parameters into magnitudes and colours of selected filter sets. The BC tables can be downloaded or implemented into large simulation projects using the interpolation code provided with the database. We computed extinction coefficients on a star-by-star basis, hence taking into account the effects of spectral type and non-linearity dependency on the total extinction. We illustrate the use of these BCs in PARSEC isochrones. We show that using spectral-type dependent extinction coefficients is necessary for Gaia filters whenever $A_{V} \gtrsim 0.5 \mathrm{mag}$. Bolometric correction tables for rotating stars and tables of limb-darkening coefficients are also provided.
\end{abstract}

Key words. Hertzsprung-Russell and C-M diagrams - astronomical databases: miscellaneous - dust, extinction open clusters and associations: individual: NGC 2425 - open clusters and associations: individual: Mellotte 22

\section{Introduction}

Bolometric corrections (BCs) are usually applied to the absolute magnitude of a star to obtain its bolometric absolute magnitude or luminosity, or conversely, to predict the magnitudes of a model star in a given set of filters. Most modern versions of BCs are usually based on theoretical stellar spectral libraries; see for example Girardi et al. (2002), Casagrande \& VandenBerg (2014, 2018a). In some cases empirical stellar spectral libraries are also used for this purpose, however, they require additional calibration from theoretical atmosphere models and are always limited by the coverage of stellar spectral type and wavelength range (Sánchez-Blázquez et al. 2006; Rayner et al. 2009; Chen et al. 2014a). In practice, BCs are often provided along with, or can be easily computed from, spectral libraries obtained from the modelling of stellar atmospheres. Currently, the widely used theoretical stellar spectral libraries include ATLAS9 (Castelli \& Kurucz 2003) and PHOENIX (Allard et al. 2012) for generic types of stars, MARCS (Gustafsson et al. 2008) for cool or intermediate temperature stars, COMARCS for $\mathrm{K} / \mathrm{M} / \mathrm{S} / \mathrm{C}$ stars (Aringer et al. 2009, 2016, 2019), and PoWR for Wolf-Rayet (WR) stars (Gräfener et al. 2002, and references

* The YBC database of stellar bolometric corrections is available at http://stev. oapd.inaf.it/YBC therein). These libraries, which have different solar abundances, offer large grids of models covering different stellar parameters (metallicity, $T_{\text {eff }}$, and $\log g$ ). However, currently there is a lack of a single, homogeneous, public database to synthesise these libraries and provide $\mathrm{BC}$ tables for a large set of photometric systems.

One of the obvious advantages of having a homogeneous database for BCs would be to facilitate the comparison between stellar models and the observations. Indeed, besides the differences in the theoretical quantities such as $\log L / L_{\odot}$ and $T_{\text {eff }}$ of various stellar models, additional deviations are often introduced from different authors using different BC tables. The possibility of testing the difference from those originally applied will become more critical with forthcoming facilities providing photometry of much higher precision. This is especially true considering, for instance, the $\sim 10^{-4} \mathrm{mag}$ errors expected for Gaia sources with $G<12 \mathrm{mag}$ and $>100$ CCD transits, for a nominal mission with perfect calibrations (see Fig. 9 in Evans et al. 2018), and the systematic errors smaller than 0.005 mag planned for Large Synoptic Survey Telescope (LSST) single visits (Ivezić et al. 2019). It is also important to consider the different ways of handling the interstellar extinction in the models, which is especially crucial when using either UV filters (as in GALEX) or very broad passbands (such as the Gaia, 
TESS, and HST/WFC3/UVIS extremely wide filters). In these cases, fixed extinction coefficients are no longer valid and the effect of stellar spectral types becomes critical (Girardi et al. 2008).

In this work, we build a database in which we assemble existing popular stellar spectral libraries to compute BC tables homogeneously for a wide variety of photometric systems. The web interface of this database provides a convenient way for the users to transform their theoretical stellar catalogues into magnitudes and colours, and hence to compare these samples with observations. This database has the flexibility to choose different stellar spectral libraries, thus allowing their differences to be easily investigated. The extinction coefficients have been computed on a star-by-star basis, therefore the variation with spectral type has been taken into account. The non-linearity as a function of $A_{V}$ is included, which is important for highly attenuated targets. In this paper, we introduce the definitions of the BCs in Sect. 2, the available stellar spectral libraries in Sect. 3, and our C/Python code package in Sect. 4. We compare some of our results with the literature in Sect. 5. In Sect. 6, we discuss the spectral type dependent extinction coefficients. Section 7 summarises the main results.

\section{Bolometric corrections}

In this section, we recall some basic relations concerning the BC, which are necessary for the discussion. Kurucz (1979), Bessell et al. (1998), and Girardi et al. (2002) provide a more exhaustive discussion.

First, we recall the definition of magnitude. Assuming we (at earth) receive a radiation flux $f_{\lambda}$ (or $f_{v}$ ) from a source, the magnitude in a certain filter band $i$ with transmission curve $S_{\lambda, i}$ is

$m_{i}=-2.5 \log \left[\frac{\int_{\lambda_{1}}^{\lambda_{2}} \lambda f_{\lambda} S_{\lambda, i} \mathrm{~d} \lambda}{\int_{\lambda_{1}}^{\lambda_{2}} \lambda f_{\lambda}^{0} S_{\lambda, i} \mathrm{~d} \lambda}\right]+m_{i, 0}$.

In this equation, $f_{\lambda}^{0}$ is the flux of the reference spectrum and $m_{i, 0}$ is the corresponding reference magnitude. These two quantities depend on the photometric system and are discussed later. The quantities $\lambda_{1}$ and $\lambda_{2}$ denote the lower and upper wavelength limits of the filter transmission curve, respectively. The above equation is valid for present-day photon-counting devices (CCDs or IR arrays). For more traditional energy-integrating systems the above equation should be changed to

$m_{i}=-2.5 \log \left[\frac{\int_{\lambda_{1}}^{\lambda_{2}} f_{\lambda} S_{\lambda, i} \mathrm{~d} \lambda}{\int_{\lambda_{1}}^{\lambda_{2}} f_{\lambda}^{0} S_{\lambda, i} \mathrm{~d} \lambda}\right]+m_{i, 0}$.

Depending on the reference spectra $f_{\lambda}^{0}$ (or $f_{v}^{0}$ ), commonly used magnitude systems are:

- Vega magnitude systems. The spectrum of Vega ( $\alpha$ Lyr) is used as the reference spectrum. The reference magnitudes are set so that Vega has a magnitude equal to, or slightly different from zero. By default, we use the latest Vega spectrum ${ }^{1}$ from the CALSPEC database (Bohlin et al. 2014).

- AB magnitude systems (Oke 1974). The reference spectrum has a constant value of $f_{v}^{0}=10^{\frac{48.60}{-2.5}} \mathrm{erg} \mathrm{s}^{-1} \mathrm{~cm}^{-2} \mathrm{~Hz}^{-1}$. The reference magnitudes thus are set to $-48.60 \mathrm{mag}$.

- Space Telescope (ST) magnitude systems (Laidler et al. 2005). The reference spectrum has a constant value of $f_{\lambda}^{0}=$ 1 Currently it is alpha_lyr_stis_008.fits from ftp://ftp.
stsci.edu/cdbs/current_calspec/ $10^{\frac{21.1}{-2.5}} \mathrm{erg} \mathrm{s}^{-1} \mathrm{~cm}^{-2} \AA^{-1}$. The reference magnitudes thus are set to $-21.10 \mathrm{mag}$.

- Gunn systems (Thuan \& Gunn 1976). In these systems, Ftype subdwarfs, in particular BD +174708 , are taken as the reference stars instead of Vega.

Among these, the Vega and AB magnitude systems are the most widely adopted ones. At http://stev.oapd.inaf.it/ $\mathrm{cmd} /$ photsys. html, the reader can check the information about the photometric systems supported.

Usually, synthetic spectral libraries provide the stellar flux at the stellar radius $R$. This flux $F_{\lambda}$ is related to the effective temperature $T_{\text {eff }}$ of the star by

$F_{\text {bol }} \equiv \int_{0}^{\infty} F_{\lambda} \mathrm{d} \lambda=\sigma T_{\mathrm{eff}}^{4}$

where $\sigma$ is the Stefan-Boltzmann constant. By placing the star at $10 \mathrm{pc}$ from the earth, the flux we receive is

$f_{\lambda, 10 \mathrm{pc}}=\left(\frac{R}{10 \mathrm{pc}}\right)^{2} F_{\lambda} 10^{-0.4 A_{\lambda}}$,

where $A_{\lambda}$ is the assumed extinction between the star and the observer. Therefore, the absolute magnitude $M_{i}$ for a photoncounting photometric system is

$$
\begin{aligned}
M_{i} & =-2.5 \log \left[\frac{\int_{\lambda_{1}}^{\lambda_{2}} \lambda f_{\lambda, 10 \mathrm{pc}} S_{\lambda, i} \mathrm{~d} \lambda}{\int_{\lambda_{1}}^{\lambda_{2}} \lambda f_{\lambda}^{0} S_{\lambda, i} \mathrm{~d} \lambda}\right]+m_{i, 0} \\
& =-2.5 \log \left[\left(\frac{R}{10 \mathrm{pc}}\right)^{2} \frac{\int_{\lambda_{1}}^{\lambda_{2}} \lambda F_{\lambda} 10^{-0.4 A_{\lambda}} S_{\lambda, i} \mathrm{~d} \lambda}{\int_{\lambda_{1}}^{\lambda_{2}} \lambda f_{\lambda}^{0} S_{\lambda, i} \mathrm{~d} \lambda}\right]+m_{i, 0} .
\end{aligned}
$$

The definition of bolometric magnitude $M_{\mathrm{bol}}$ is

$$
\begin{aligned}
M_{\mathrm{bol}} & =M_{\mathrm{bol}, \odot}-2.5 \log \left(L / L_{\odot}\right) \\
& =M_{\mathrm{bol}, \odot}-2.5 \log \left(4 \pi R^{2} F_{\mathrm{bol}} / L_{\odot}\right) .
\end{aligned}
$$

According to the IAU 2015 resolution (Mamajek et al. 2015), the absolute bolometric magnitude for the nominal solar luminosity $\left(3.828 \times 10^{26} \mathrm{~W}\right)$ is $M_{\mathrm{bol}, \odot}=4.74 \mathrm{mag}$.

Given an absolute magnitude $M_{i}$ in a given filter band $i$ for a star of absolute bolometric magnitude $M_{\mathrm{bol}}$, the bolometric correction $\mathrm{BC}_{i}$ is:

$\mathrm{BC}_{i}=M_{\mathrm{bol}}-M_{i}$

By combing Eqs. (4)-(6), we have

$$
\begin{aligned}
\mathrm{BC}_{i}= & M_{\mathrm{bol}, \odot}-2.5 \log \left(\frac{4 \pi \sigma(10 \mathrm{pc})^{2}}{L_{\odot}}\right)-2.5 \log \left(T_{\mathrm{eff}}^{4}\right) \\
& +2.5 \log \left[\frac{\int_{\lambda_{1}}^{\lambda_{2}} \lambda F_{\lambda} 10^{-0.4 A_{\lambda}} S_{\lambda, i} \mathrm{~d} \lambda}{\int_{\lambda_{1}}^{\lambda_{2}} \lambda f_{\lambda}^{0} S_{\lambda, i} \mathrm{~d} \lambda}\right]-m_{i, 0} .
\end{aligned}
$$

The advantage of using the above equation to compute BCs is that the stellar radius $R$ disappears. In some cases, the stellar spectral library is computed in plane-parallel geometry and there is no definition of a geometrical stellar radius (but the optical depth). Therefore, once $F_{\lambda}$ (related to $T_{\text {eff }}$ by Eq. (3)) for a star with given $T_{\text {eff }}, \log g$ and metallicity $[\mathrm{M} / \mathrm{H}]$ is provided, the corresponding $\mathrm{BC}_{i}$ can be derived. 
The above definition of the BC is valid for present-day photon-counting devices (CCDs or IR arrays), while for energyintegrating systems the above equation should be changed to

$$
\begin{aligned}
\mathrm{BC}_{i}= & M_{\mathrm{bol}, \odot}-2.5 \log \left(\frac{4 \pi \sigma(10 \mathrm{pc})^{2}}{L_{\odot}}\right)-2.5 \log \left(T_{\mathrm{eff}}^{4}\right) \\
& +2.5 \log \left[\frac{\int_{\lambda_{1}}^{\lambda_{2}} F_{\lambda} 10^{-0.4 A_{\lambda}} S_{\lambda, i} \mathrm{~d} \lambda}{\int_{\lambda_{1}}^{\lambda_{2}} f_{\lambda}^{0} S_{\lambda, i} \mathrm{~d} \lambda}\right]-m_{i, 0} .
\end{aligned}
$$

For $\mathrm{AB}$ magnitude systems with photon-counting devices, we can either convert $f_{v}^{0}$ to $f_{\lambda}^{0}$ and use Eq. (7), or use the following equation instead:

$$
\begin{aligned}
\mathrm{BC}_{i}= & M_{\mathrm{bol}, \odot}-2.5 \log \left(\frac{4 \pi \sigma(10 \mathrm{pc})^{2}}{L_{\odot}}\right)-2.5 \log \left(T_{\text {eff }}^{4}\right) \\
& +2.5 \log \left[\frac{\int_{v_{1}}^{v_{2}} F_{v} 10^{-0.4 A_{v}} S_{v, i} \mathrm{~d} v / v}{\int_{v_{1}}^{v_{2}} f_{v}^{0} S_{v, i} \mathrm{~d} v / v}\right]-m_{i, 0} .
\end{aligned}
$$

Similarly, the equation for AB magnitude system with energyintegrating devices is

$$
\begin{aligned}
\mathrm{BC}_{i}= & M_{\mathrm{bol}, \odot}-2.5 \log \left(\frac{4 \pi \sigma(10 \mathrm{pc})^{2}}{L_{\odot}}\right)-2.5 \log \left(T_{\mathrm{eff}}^{4}\right) \\
& +2.5 \log \left[\frac{\int_{v_{1}}^{v_{2}} F_{v} 10^{-0.4 A_{\nu}} S_{v, i} \mathrm{~d} v}{\int_{v_{1}}^{v_{2}} f_{v}^{0} S_{v, i} \mathrm{~d} v}\right]-m_{i, 0} .
\end{aligned}
$$

\section{Stellar spectral libraries}

In this section, we briefly describe the stellar spectral libraries currently supported in our database. We will continuously expand it with data from external sources or provided by our group.

\subsection{ATLAS9 models}

One of the most widely used stellar spectral libraries is the set of plane parallel models computed by Castelli \& Kurucz $(2003)^{2}$ with the ATLAS9 code (Kurucz 2014). These models are based on the solar abundances by Grevesse \& Sauval (1998) and make use of an improved set of molecular absorption lines including $\mathrm{TiO}$ and $\mathrm{H}_{2} \mathrm{O}$, as well as absorption lines from quasi-molecular $\mathrm{H}-\mathrm{H}$ and $\mathrm{H}-\mathrm{H}^{+}$. The model grids are computed for $T_{\text {eff }}$ from $3500 \mathrm{~K}$ to $50000 \mathrm{~K}, \log g$ ( $g$ in cgs unit) from 0.0 dex to $5.0 \mathrm{dex}$, and $[\mathrm{M} / \mathrm{H}]=+0.5,+0.2,0.0,-0.5,-1.0,-1.5,-2.0,-2.5,-3.5$, -4 and -5.5 .

\subsection{PHOENIX models}

The PHOENIX database (Allard \& Hauschildt 1995; Allard et al. 1997 ) is another widely used stellar spectra library, especially for cool stars. The atmospheres of cool stars are dominated by molecular formation and by dust condensation at very low temperature. Both of these two phenomena can significantly affect the spectral shape. A suitable set of $1 \mathrm{D}$, static spherical atmosphere spectral models accounting for the above effects is provided in Allard et al. (2012). Among the different model suites of this database, we use the BT-Settl ${ }^{3}$ models for the coverage completeness in stellar parameters and for the wide usage

\footnotetext{
2 http://wwwuser.oats. inaf.it/castelli/grids.html

3 https://phoenix.ens-lyon.fr/Grids/BT-Settl/ AGSS2009/
}

in the literature. The BT-Settl models use the Asplund et al. (2009) solar abundances. These abundances are provided for $2600 \mathrm{~K} \leq T_{\text {eff }}<50000 \mathrm{~K}, 0.5<\log g<6$, and metallicities $-4.0 \lesssim[\mathrm{M} / \mathrm{H}] \lesssim+0.5$.

\subsection{WM-basic models}

For temperatures typical of $\mathrm{O}$ and $\mathrm{B}$ stars $(19000 \mathrm{~K}<$ $T_{\text {eff }}<60000 \mathrm{~K}$ ) we computed a library of models using the public code WM-basic (Pauldrach et al. 1986), as described in Chen et al. (2015). This allows us to consider both the effects of extended winds and those of non-LTE because both effects may significantly affect the emergent spectra of hot stars. These models use the solar abundances of PARSEC (Bressan et al. 2012), which compiled the results from Grevesse \& Sauval (1998), Caffau et al. (2011) and references therein. The model grids are computed for metallicities of $Z=0.0001,0.0005,0.001,0.004,0.008$, and 0.02 . The value $\log T_{\text {eff }}$ covers the interval from 4.3 to 5 with a step of $0.025 \mathrm{dex}$, while $\log g$ goes from 2.5 to 6.0 with a step of $0.5 \mathrm{dex}$. For each $\log T_{\text {eff }}$ and $\log g$, models with three values of mass loss rate $\left(\dot{M}=10^{-7}, 10^{-6}\right.$, and $\left.10^{-5} M_{\odot} \mathrm{yr}^{-1}\right)$ are computed if convergence is reached for them, as detailed in Chen et al. (2015).

\subsection{PoWR models}

Wolf-Rayet stars typically have wind densities one order of magnitude larger than those of massive O-type stars. Spectroscopically these WR stars are dominated by the presence of strong broad emission lines of helium, nitrogen, carbon, and oxygen. They are subdivided into different subtypes: one with strong lines of helium and nitrogen (WN stars) another with strong lines of helium and carbon (WC stars), and a third with strong oxygen lines (WO stars). The PoWR ${ }^{4}$ group has provided models for WR stars (Gräfener et al. 2002; Hamann \& Gräfener 2004; Sander et al. 2012; Todt et al. 2015). These models adopt the solar abundances from Grevesse \& Sauval (1998), and are computed for Milky Way, Large Magellanic Cloud (LMC), Small Magellanic Cloud (SMC), and sub-SMC metallicities. For each metallicity, late-type WN stars of different hydrogen fraction, early-type WN stars, and WC (except for SMC and sub-SMC metallicities at the moment) stars are computed. For a given spectral type and metallicity, the models are computed as a function of $\log T_{\text {eff }}$ and the transformed radius $\log R_{\mathrm{t}}$ (Schmutz et al. 1989), which is a more important quantity than $\log g$ in these models dominated by stellar winds. The coverage in $\log T_{\text {eff }}$ ( 4.5-5.2) and $\log R_{\mathrm{t}}(\sim 0 .-1.6)$ changes with metallicity and spectral type. The transformed radius is defined as

$R_{\mathrm{t}}=R_{*}\left(\frac{v_{\infty}}{2500 \mathrm{~km} \mathrm{~s}^{-1}} / \frac{\dot{M}}{10^{-4} M_{\odot} \mathrm{yr}^{-1}}\right)^{2 / 3}$,

where $R_{*}, v_{\infty}$, and $\dot{M}$ are the hydro-static stellar radius, terminal velocity, and mass loss rate, respectively. We note that in the original definition of $R_{\mathrm{t}}$ of PoWR, a multiplication factor $D^{-1 / 3}$ is applied to the above equation, where $D$ is the clumping factor. For different types of WR models of PoWR, different clumping factors have been used. To facilitate the interpolation, we divided the $R_{\mathrm{t}}$ values from PoWR by this factor, therefore it disappears in the above equation.

\footnotetext{
4 http://www.astro.physik.uni-potsdam.de/ wrh/PoWR/ powrgrid1.php
} 


\subsection{COMARCS models}

The local thermodynamic equilibrium (LTE) and chemical equilibrium without dust, spherical symmetric, hydrostatic COMARCS $^{5,6}$ database provides models for C-, S-, K-, and Mtype stars for modelling TP-AGB stars and other red giants (Aringer et al. 2009, 2016, 2019). The solar chemical composition is based on Caffau et al. (2009a,b). The carbon star grids cover a metallicity $Z$ range from 0.001 to $0.016, T_{\text {eff }}$ from $2500 \mathrm{~K}$ to $4000 \mathrm{~K}$, and $\log g$ from -1 to 2 , while the $\mathrm{K}$ and $\mathrm{M}$ star grids cover a metallicity range from 0.00016 to $0.14, T_{\text {eff from } 2600 \mathrm{~K}}$ to $4800 \mathrm{~K}$, and $\log g$ from -1 to 5 .

\subsection{DA white dwarf spectral libraries}

Gaia DR2 parallaxes (Luri et al. 2018) allowed the placement of a significant number of white dwarfs into absolute magnitude versus colour diagrams, hence ensuring a wide interest in including these objects in isochrone-fitting methods. Therefore we include the Koester (2010) and Tremblay \& Bergeron (2009) DA white dwarf libraries (downloaded from ${ }^{7}$ ) in our database. In these plane-parallel models LTE and hydrostatic equilibrium are assumed. The library covers the $T_{\text {eff }}$ range from $5000 \mathrm{~K}$ to $80000 \mathrm{~K}$ and $\log g$ ranges from 6.5 to 9.5 .

\subsection{ATLAS12 models with $\alpha$-enhancement}

To study the photometric properties of alpha-enhanced PARSEC stellar models, we computed atmosphere models with the latest ATLAS12 code $^{8}$ with an updated line lists. These models have been used in Fu et al. (2018) and have been shown to improve the fitting to the galactic globular cluster 47 Tuc observations with HST ACS/WFC3. For the moment, we only computed these spectra for two metallicities corresponding to two 47 Tuc stellar populations (Fu et al. 2018), however, work is in progress to extend them to other metallicities together with the PARSEC alpha-enhanced stellar tracks. These models cover $T_{\text {eff }}$ from $4000 \mathrm{~K}$ to $21000 \mathrm{~K}$, and $\log g$ from 0.5 to 5 . Below $10000 \mathrm{~K}$, the step in $T_{\text {eff }}$ is $100 \mathrm{~K}$, while above the step is $200 \mathrm{~K}$. The step in $\log g$ is 0.5 dex.

\subsection{Spectra of rotating stars based on Kurucz models}

In Girardi et al. (2019), we computed a spectral library for rotating stars with a new approach. This approach takes into account the limb-darkening effect and that of the stellar surface effective temperature variation due to rotation, which results in the photometric differences when the star is observed from different angles. This library is based on the spectral intensity library from Kurucz website ${ }^{9}$. The Kurucz models cover $T_{\text {eff }}$ from $3500 \mathrm{~K}$ to $50000 \mathrm{~K}, \log g$ from 0 to 5 , and $[\mathrm{M} / \mathrm{H}]$ from -5 to +1 . The wavelength coverage is the same as that of ATLAS9 models, from $90.9 \AA$ to $16 \mu \mathrm{m}$.

\subsection{Tables of limb-darkening coefficients}

The limb-darkening effect is important to analyse light curves of eclipsing binaries and microlensing events, and for probing

\footnotetext{
5 http://stev.oapd.inaf.it/atm

6 http://stev.oapd.inaf.it/synphot/Cstars

7 http://svo2.cab.inta-csic.es/theory/newov/

8 https://www.oact.inaf.it/castelli/castelli/sources/ atlas $12 . \mathrm{html}$

9 http://kurucz.harvard.edu/
}

exoplanet atmospheres and spatially resolved stellar surfaces. Accurate empirical determination of limb-darkening coefficients through eclipsing binaries observations is not possible yet, mainly because of parameter degeneracies. Therefore, stellar atmosphere models are essential in these studies. Based on the spectral intensity library from the Kurucz website, we computed the specific intensity $I(\mu)$ as a function of $\mu=\cos \theta$, where $\theta$ is the angle between the line of sight and the surface normal for each of our filter systems. Specific intensities have been computed for both photon-counting and energy-integrating detectors, respectively, as

$I_{i}(\mu)=\frac{\int_{\lambda} I_{\lambda}(\mu) S_{\lambda, i} \lambda \mathrm{d} \lambda}{\int_{\lambda} S_{\lambda, i} \lambda \mathrm{d} \lambda}$

and

$I_{i}(\mu)=\frac{\int_{\lambda} I_{\lambda}(\mu) S_{\lambda, i} \mathrm{~d} \lambda}{\int_{\lambda} S_{\lambda, i} \mathrm{~d} \lambda}$.

We further computed the limb-darkening coefficients $\left(a_{1}, a_{2}, a_{3}\right.$, and $a_{4}$ ) with the following fitting relation proposed by Claret (2000):

$\frac{I(\mu)}{I(1)}=1-\sum_{n=1}^{4} a_{n}\left(1-\mu^{n / 2}\right)$.

These coefficients are provided mainly for a question of completeness. Indeed, they are computed homogeneously for all filter sets included in our database.

\subsection{Stellar spectral library selection}

The above-described libraries can be used either combined or separately. The default library selection scheme is as follows:

(1) ATLAS9 is used when $T_{\text {eff }}>6500 \mathrm{~K}$.

(2) PHOENIX is used when $T_{\text {eff }}<5500 \mathrm{~K}$.

(3) A smooth interpolation between the previous two when $\geq T_{\text {eff }} \leq 6500 \mathrm{~K}$.

(4) COMARCS $\mathrm{M} / \mathrm{S}$ star library is used when $\left(X_{\mathrm{C}} / 12\right) /\left(X_{\mathrm{O}} / 16\right) \leq 1$ and $T_{\mathrm{eff}}<4850 \mathrm{~K}$ and $\log g>1.5$.

(5) A smooth interpolation between PHOENIX and COMARCS $\mathrm{M} / \mathrm{S}$ star libraries exist in the overlapping region between them.

(6) COMARCS $C$ star library is used when $\left(X_{\mathrm{C}} / 12\right) /\left(X_{\mathrm{O}} / 16\right)>1$ and $T_{\text {eff }}<4500 \mathrm{~K}$ and $\log g<2.5$.

(7) WM-basic libraries are used when $T_{\text {eff }}>20000 \mathrm{~K}$ and the mass loss rate $\dot{M}>10^{-8} M_{\odot} \mathrm{yr}^{-1}$.

(8) PoWR libraries are used when $T_{\text {eff }}>100000 \mathrm{~K}$ or $X<$ $0.8 X_{\mathrm{i}}$ or $X<0.65$, where $X_{\mathrm{i}}$ represents the original surface hydrogen mass fraction.

(9) PoWR WC library is used when PoWR libraries are selected and $X_{\mathrm{C}} / 12>X_{\mathrm{N}} / 14$ and $X_{\mathrm{C}}>0.05$.

(10) Koester WD library is used when $\log g>6$ and $T_{\text {eff }}>$ $6300 \mathrm{~K}$.

The stellar surface chemical compositions are used in the above scheme, therefore they are required in the web interface (see Appendix A for more details). If the required chemical abundances are not provided by the user, a solar scaled abundance is used with the specified metallicity $Z$, which means all the relevant abundance ratios are the same as those in the Sun. As a test case we applied the new BCs to the PARSEC V1.2s isochrones. In Fig. 1, we show the isochrones with $[\mathrm{M} / \mathrm{H}]=0$ and for selected ages in the standardised $J K$ system from 

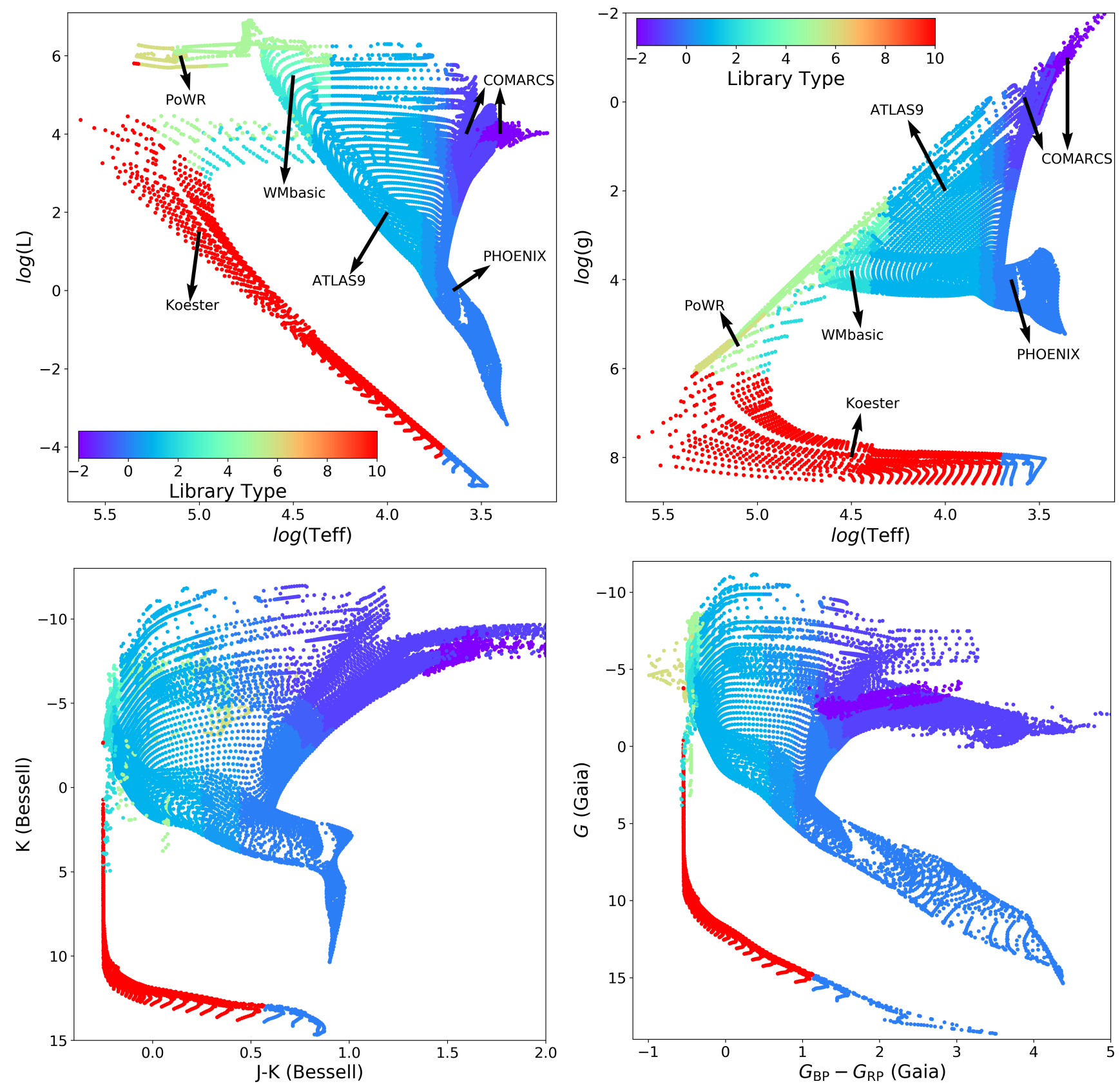

Fig. 1. PARSEC $v 1.2 S$ isochrones of $[\mathrm{M} / \mathrm{H}]=0.0$ and ages from $\log (\mathrm{age} / \mathrm{yr})=6.6-10.1$ complete from pre-main sequence to white dwarf stages, or up to the carbon-ignition in the case of massive stars. Upper left: Hertzsprung-Russell diagram $\log L / L_{\odot}$ vs. $\log T_{\text {eff }}$ diagram; upper right: Kiel diagram $\log g$ vs. $\log T_{\text {eff }}$; lower left: CMD in Bessell $J K$; lower right: CMD in Gaia DR2 filters. The "library type" we assign to different libraries are -2 for COMARCS C star library, -1 for COMARCS M/S star library, 0 for PHOENIX library, 1 for ATLAS9 or ATLAS12 library, 2 for WM-basic library with $\dot{M}=10^{-7} M_{\odot} \mathrm{yr}^{-1}, 3$ for WM-basic library with $\dot{M}=10^{-6} M_{\odot} \mathrm{yr}^{-1}, 4$ for WM-basic library with $\dot{M}=10^{-5} M_{\odot} \mathrm{yr}^{-1}, 5$ for PoWR WNL and WNE libraries, 6 for PoWR WC library, and 10 for Koester WD library. If a star is interpolated between different libraries, an intermediate number is assigned. However, in the future these numeric labels might be changed if more libraries are implemented or the interpolation scheme is changed.

Bessell (1990) and in the Gaia DR2 passband as described in Maíz Apellániz \& Weiler (2018); the Gaia filters used in the following sections are also from this reference, unless specified otherwise. Different colours indicate the different stellar spectral libraries adopted, as specified in the caption.

Aside from the above default scheme, we also offer some other options, such as ATLAS9 only, PHOENIX only, or a combination of these (with an adjustable transition region). An issue concerns the difference in broadband color-magnitude diagrams
(CMDs) between the different libraries. We take the comparison between ATLAS9 and PHOENIX as an example to address this issue. In Fig. 2 we show the differences between PHOENIX and ATLAS9 libraries in several CMDs, using the same PARSEC $\mathrm{v} 1.2 \mathrm{~S}$ isochrones of $[\mathrm{M} / \mathrm{H}]=0.0$ and ages $\log (\mathrm{age} / \mathrm{yr})=8,9$, and 10. The difference between PHOENIX and ATLAS9 increases at decreasing $T_{\text {eff }}$. At the solar metallicity, the colour difference between these two atmosphere models can reach $\sim 0.05$ mag at the main-sequence turn-off and $\sim 0.1 \mathrm{mag}$ at the giant branch 

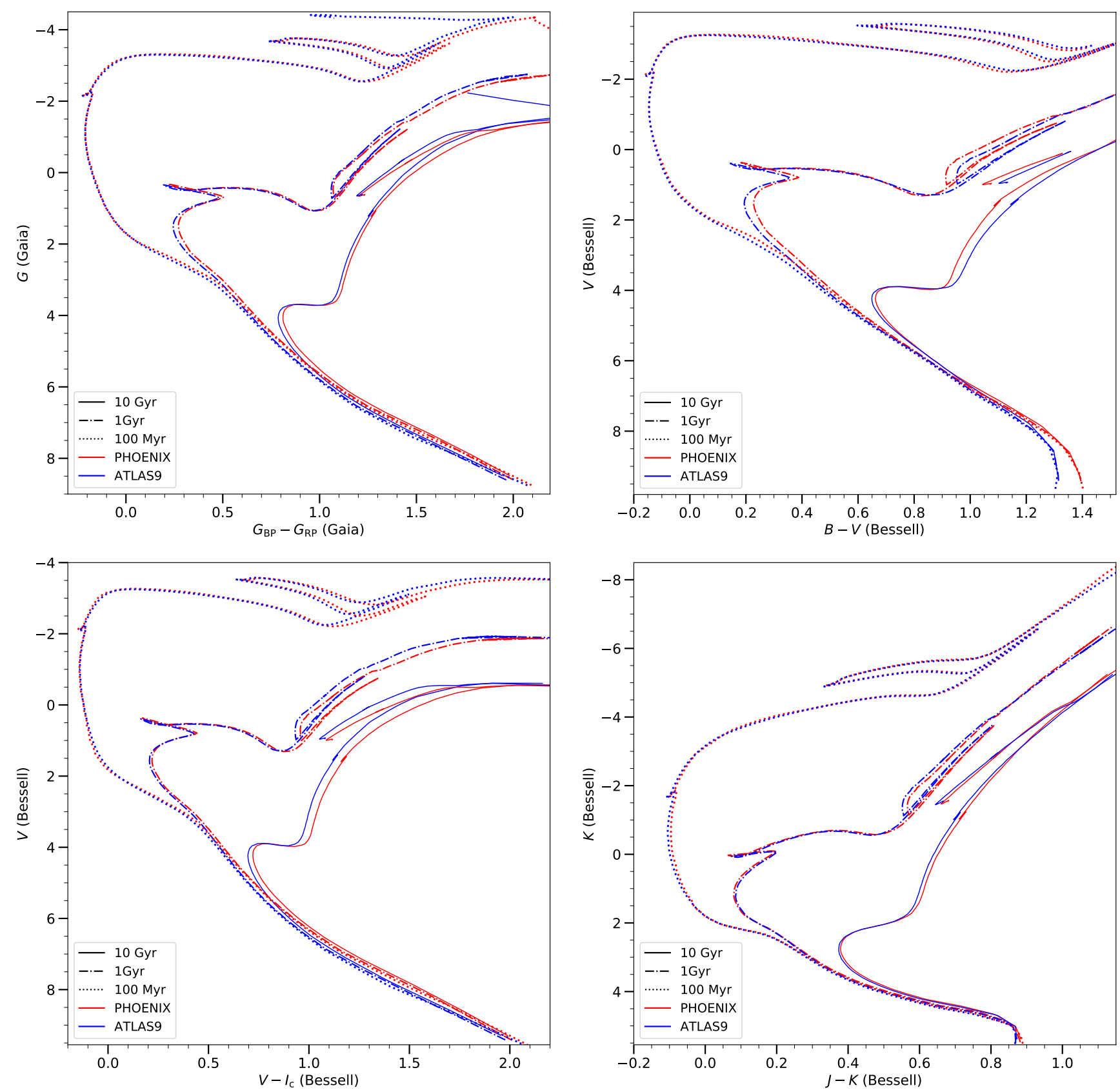

Fig. 2. Differences between PHOENIX and ATLAS9 models in the CMDs. From left to right and top to bottom: Gaia $G_{\mathrm{BP}}-G_{\mathrm{RP}} \mathrm{vs.} G, B-V$ vs. $V$, $V-I_{\mathrm{c}}$ vs. $V$ and $J-K$ vs. $K$. The Gaia filters are from Maíz Apellániz \& Weiler (2018), while the standard filters are from Bessell \& Brett (1988), Bessell (1990). PARSEC v1.2S isochrones of $[\mathrm{M} / \mathrm{H}]=0.0$ and ages $\log (\mathrm{age} / \mathrm{yr})=8,9$, and 10 are plotted. The ATLAS9 models are only calculated for stars hotter than above $3500 \mathrm{~K}$, therefore those parts of the isochrones with $T_{\text {eff }}$ below $3500 \mathrm{~K}$ are not shown.

in the $V-(V-I)$ plane. For the lower main-sequence stars, we showed in our previous work (Chen et al. 2014b) that PHEONIX offers a better fitting compared to ATLAS9. Moreover, the PHOENIX library is computed with spherical geometry, making it preferable than ATLAS9 models especially for giants.

\section{Code package and web interface}

The design of this package is to make it transparent to the user and extensible to adopting new stellar spectral libraries, filter sets, and definitions of photometric systems. The whole package is divided into three steps. In the first step, the original stellar spectral libraries are assembled and re-sampled to FITS/HDF5 format files. In the second step, these files are used to compute
BCs. In the third step, these BC tables are used in interpolation for stars of given stellar parameters. The code for the first and second steps is written in Python because the computational speed is not an issue and we only need to compute those tables once for all. Compared to $\mathrm{C}$ or Fortran, Python provides highlevel libraries for manipulating tabular data (including ASCII, FITS, and HDF5 formats). The third step is written in C language for a faster computational speed.

\subsection{Assembled FITS/HDF5 files of spectra}

In this step, the original spectra are assembled into FITS (Wells et al. 1981; Pence 1999) and/or HDF5 (Koziol \& Robinson 2018) format files. If the original spectra are in very high resolution, 
i.e. the PHOENIX models contain $\sim 23000$ wavelength points which are not necessary for the $\mathrm{BC}$ calculation other than narrow-band filters; these are re-sampled into a lower resolution wavelength grid before assembling for computational speed and file size. The re-sampling is done with a modified version of SpectRes package (Carnall 2017), which preserves the integrated flux within each of the wavelength grids. After this step, the stellar spectral libraries are stored in a homogeneous file format and organisation. This step is necessary as the original stellar spectral libraries are provided in different formats. For example, the PHOENIX models are stored in a single ASCII file for each spectrum, while ATLAS9 models are stored in one single ASCII file for all the models of a given metallicity. Currently, the assembled spectra for non-rotating stars are stored in FITS format and can be easily viewed with TOPCAT (Taylor 2017), while the assembled spectra for rotating stars are stored in HDF5 format for their logical complexity.

\subsection{Computing tables of BCs}

In this step, the above-generated FITS format spectra are read to compute the BCs, for different filter sets available, according to Eqs. (7)-(10). The BCs are first computed for the original grids. However, these original grids are usually not rectangular or uniformly distributed in the $\log T_{\text {eff }}-\log g\left(\log R_{\mathrm{t}}\right)$ plane. For example, at high $\log T_{\text {eff }}$, usually the high $\log g$ models are missing owing to the proximity to the Eddington limit. We always extend the grids in $\log g$ with neighbouring models of the same $\log T_{\text {eff }}$. Thereafter, these rectangular grids are re-sampled in $\log T_{\text {eff- }}-$ $\log g\left(\log R_{\mathrm{t}}\right)$ plane with fixed steps. This re-sampling enables fast index searching for the interpolation when utilising the $\mathrm{BC}$ tables. These final $\mathrm{BC}$ tables are stored into FITS format files. The BCs with different $A_{V}$ s are assembled in to the same file, therefore it is possible to easily check some of the discussions presented in Sect. 6 (such as Fig. 4). Furthermore, this Python package provides an option to use "gnu-parallel" tool (Tange 2011) for computing tables for many different filter sets in parallel.

\subsection{Interpolation scheme}

After the BC tables are computed, users can use these tables with their own interpolation code or we can provide a $\mathrm{C}$ code upon request. In summary, this code employs linear interpolations in $\log T_{\text {eff }}, \log g$ or $\log R_{\mathrm{t}}, \log \dot{M}$, and $\log Z$. First, the BC tables in a specified filter set are loaded into the memory. An input star is then assigned a label according to its $\log T_{\text {eff }}$ and $\log g$ (or $\log R_{\mathrm{t}}$ for WRs), or also spectral type in the case of WRs. This label tells the code to which libraries are assigned. If this star is in the transition region between two libraries, both libraries are selected. A weight is given according to the proximity of the star to each library. Within a library, the interpolation is done in the $\log T_{\text {eff }}$ and $\log g$ grids of two neighbouring metallicities and then between the two metallicities. Because the $\log T_{\text {eff }}$ and $\log g$ grids are pre-sampled at fixed grids, the interpolation in the $\log T_{\text {eff }}$ and $\log g$ plane is very fast. For instance, with a computer of $3.6 \mathrm{GHz} \mathrm{CPU}$, it takes less than $20 \mathrm{~s}$ for a catalogue of $10^{6}$ stars in the standard UBVRIJHK filters. The memory consumption is less than $20 \mathrm{Mby}$.

In the interpolation, there are issues concerning the metallicities used in different stellar spectral libraries. Different libraries were computed with different solar abundances and different $\alpha$-enhancement. Part of the difference presented in the Fig. 2 comes from the metallicity difference. Moreover, the isochrones or theoretical stars may have solar abundances and $\alpha$-enhancement different from the stellar atmosphere models. Currently, we only consider the total metallicities $(Z)$ for each of the libraries. The total metallicities are computed with their own solar abundances. Although different solar abundances and $\alpha$-enhancements affect the detailed spectral features, the broadband BCs are less affected and the interpolation in the total metallicities can be taken as the first-order approximation. We recorded the solar abundances and $\alpha$-enhancements information in the code and may consider these effects when a better interpolation scheme is defined. We are also computing atmosphere models with the same abundances as used for computing PARSEC tracks, with the ATLAS12 code. These models will ensure the consistency between the atmosphere models and PARSEC stellar models, and may also allow us to evaluate the above issues.

\subsection{Web interface}

We built an easy-to-use web interface $\left(\mathrm{YBC}^{10}\right)$ for the users to convert directly any uploaded catalogue containing theoretical quantities into magnitudes and colours (not necessarily the PARSEC isochrones). More details about the web interface are provided in Appendix A. The above assembled spectral libraries, as well as the BC tables in different filters, can be downloaded at the "Spectral libraries" and the "BC tables" sections of this website, respectively.

\section{Comparison with literature results}

In this section, we compare our BCs derived from ATLAS9 models with those from some well-known studies on temperature scales or BCs. This is intended mainly as a general consistency check, rather than a detailed comparison.

Ramírez \& Meléndez (2005) provided empirical temperature scales for stars with $4000 \lesssim T_{\text {eff }} \lesssim 7000 \mathrm{~K}$ and $-3.5 \lesssim$ $[\mathrm{Fe} / \mathrm{H}] \lesssim 0.4$. In the upper panels of Fig. 3, we compare their $V-K_{\mathrm{s}}$ and $V-I_{\mathrm{c}}$ vs. $T_{\text {eff }}$ relations with ours. They are consistent to within $\sim 0.1 \mathrm{mag}$. We also plot the empirical relations for the dwarfs and sub-giants from Casagrande et al. (2010), which improve the comparison with the theoretical values for the relatively hot stars, but behave oppositely for cooler stars. This may indicate that there are still some large uncertainties in determining the physical parameters for cool stars. Casagrande \& VandenBerg (2014, 2018a,b, denoted as Casagrande in the figure) presented a code to compute the BCs based on the MARCS (Gustafsson et al. 2008) atmosphere models. In the middle panels of Fig. 3, we show the comparison for both the $V-K_{\mathrm{s}}$ and $G_{\mathrm{BP}}-G_{\mathrm{RP}}$ colours. We find a very good agreement between ours and theirs. Finally, in the bottom panels we compare our BCs with those based on 3D atmosphere models from Chiavassa et al. (2018). The agreement is also very reasonable, except for some departure at low $T_{\text {eff }}$. This might implicate that the atmospheres of cool stars could be better modelled with 3D models.

The above comparisons indicate that there is a general agreement between theoretical BCs derived from different 1D models or 3D models (except for cool stars). There is however some nonnegligible difference between $1 \mathrm{D}$ models and empirical relations or 3D relations, especially for cool stars, which deserves further investigation to solve the discrepancies.

${ }^{10}$ http://stev.oapd.inaf.it/YBC 

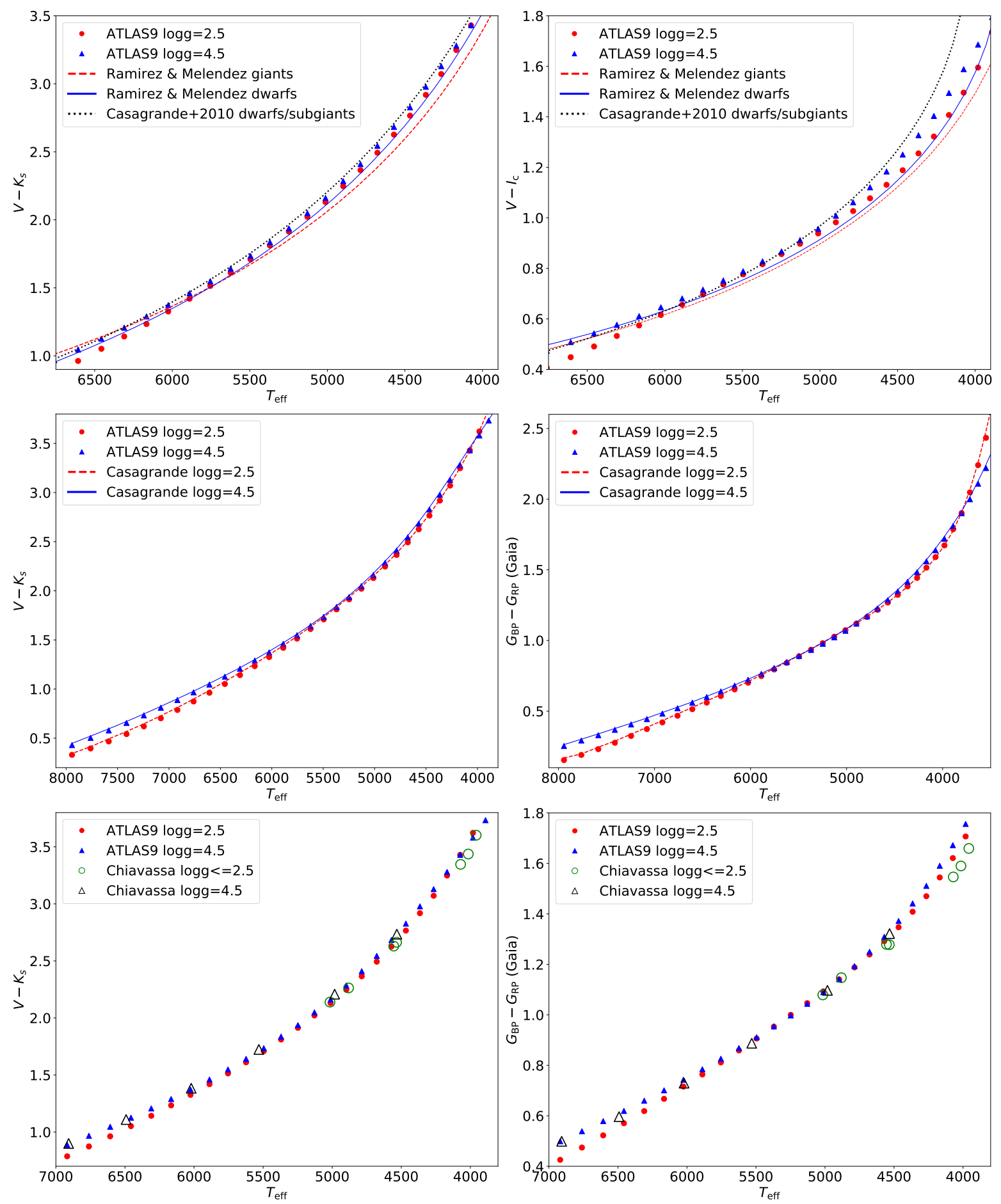

Fig. 3. Comparison of temperature scales for $[\mathrm{Fe} / \mathrm{H}]=0$ for standard filters (Bessell 1990; Maíz Apellániz 2006), 2MASS (Cohen et al. 2003), and Gaia filters. Upper panels: comparison with the empirical temperature scales from Ramírez \& Meléndez (2005) (blue solid and red dashed lines) and Casagrande et al. (2010) (black dotted lines). Middle panels: comparison with the results from Casagrande \& VandenBerg (2014, 2018a,b); the Gaia filters are from Evans et al. (2018). Lower panels: comparison with the 3D models from Chiavassa et al. (2018); the Gaia filters are those from Jordi et al. (2010). Our BCs are derived from ATLAS9 models.

\section{Spectral type dependent extinction coefficients}

Interstellar extinction is included into Eqs. (7) and (8) using extinction curves computed with the "extinction"11 Python routine, which includes most of the popular extinction laws in the literature. By default, our database is computed using

\footnotetext{
11 https://extinction. readthedocs.io
}

Cardelli et al. (1989) plus the O'Donnell (1994) extinction law (CCM+O94) with $R_{V}=3.1$, to maintain consistency with the CMD web page. However, other extinction laws with different $R_{V}$ can be computed upon request. For example, we also include the extinction curve from Wang \& Chen (2019) (priv. comm.), in which they found a non-negligible discrepancy with that of Cardelli et al. (1989) along our Galaxy by using a large data set of photometry from Gaia DR2 and other 

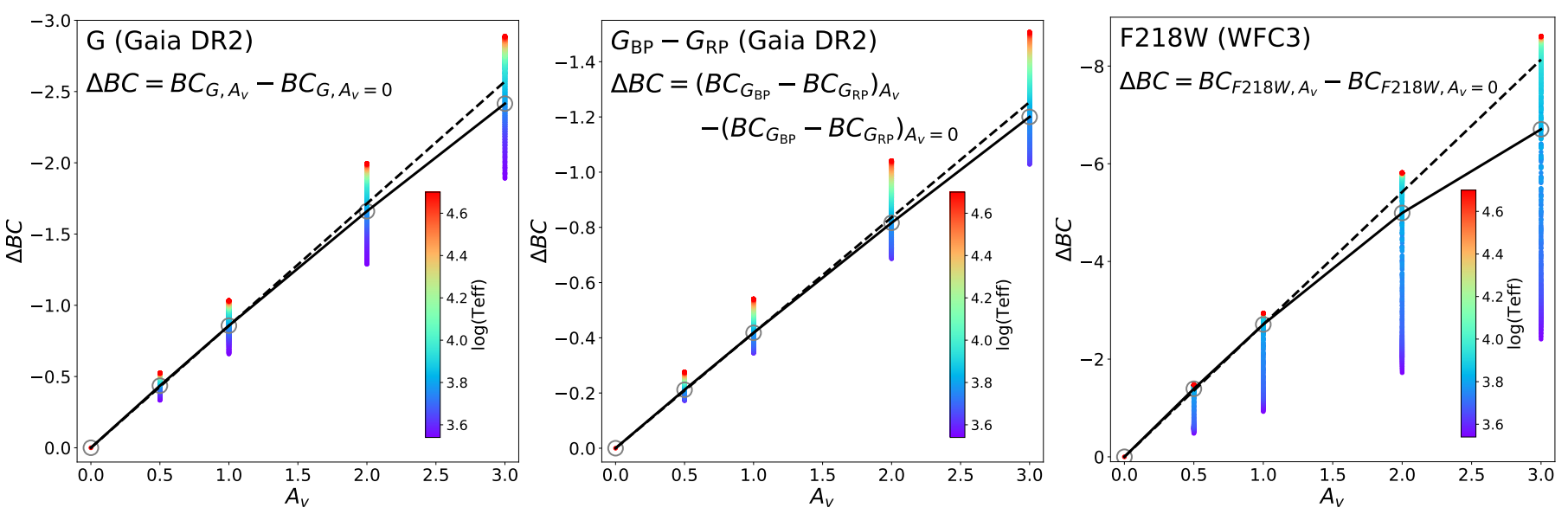

Fig. 4. $\triangle \mathrm{BC}=\mathrm{BC}_{A_{V}}-\mathrm{BC}_{A_{V}=0}$ as a function of $T_{\mathrm{eff}}$ for Gaia $G$ band (left panel), Gaia $G_{\mathrm{BP}}-G_{\mathrm{RP}}$ colour (central panel), and the WFC3/UVIS F218W filter (right panel). The grey open circles denote the models with fixed extinction coefficients at the filter centres, which are connected by the solid lines. The dashed lines indicate a linear extrapolation of the open circles of $A_{V}=0$ and $A_{V}=1$ mag, for the same $T_{\text {eff }}$. The ATLAS9 models are used in this figure, but the PHOENIX models give consistent results.

surveys. In the following discussion, if not specified, $\mathrm{CCM}+\mathrm{O} 94$ is used.

The effect of extinction on each passband can be easily evaluated by looking at the quantity $\triangle \mathrm{BC}=\mathrm{BC}_{A_{V}}-\mathrm{BC}_{A_{V}=0}$. For very narrow filters or for hypothetical monochromatic sources, the relationship between $\triangle \mathrm{BC}$ and $A_{V}$ would be linear. However, in the general case of a broad filter, where the stellar flux can change significantly within the filter wavelength range, the quantity $\triangle \mathrm{BC}$ varies as functions of spectral type and total $A_{V}$. This can be seen in Fig. 4, where we plot $\triangle \mathrm{BC}$ as a function of $A_{V}(=1$, 2, and $3 \mathrm{mag}$ ) for the Gaia $G$ band (Maíz Apellániz \& Weiler 2018), the Gaia $G_{\mathrm{BP}}-G_{\mathrm{RP}}$ colour, and the WFC3/UVIS F218W magnitude (also see Girardi et al. 2008, for ACS filters), using the ATLAS9 spectral library. The grey open circles denote the models with fixed extinction coefficients at the filter centres, which are connected by the solid lines. At every $A_{V}$ the dispersion is caused by different $T_{\text {eff }}$ and to a lesser extent also by $\log g$ (which cannot be seen in this figure, but is clear when checking the $\mathrm{BC}$ tables). At $A_{V}=0.5 \mathrm{mag}$, there is already a $\sim 0.2 \mathrm{mag}$ difference in the Gaia $G$ band, $\sim 0.1 \mathrm{mag}$ in the Gaia $G_{\mathrm{BP}}-G_{\mathrm{RP}}$ colour, and $\sim 1 \mathrm{mag}$ in the F $218 \mathrm{~W}$ filter. Therefore, we suggest using spectral-type dependent extinction coefficients for Gaia filters and UV filters whenever $A_{V} \gtrsim 0.5 \mathrm{mag}$. This dispersion increases significantly with increasing $A_{V}$. At $A_{V}=3 \mathrm{mag}$, for instance, the dispersion is about 1 mag for the Gaia $G$ band. This means that spectral type dependent extinction coefficients are very necessary, especially at large $A_{V}$. Qualitatively speaking, fixed extinction coefficients would make hot stars bluer and cool stars redder compared to the case with variable extinction coefficients.

Furthermore, for a given spectrum and filter band, $\triangle \mathrm{BC}$ is not a linear function of $A_{V}$. Figure 4 shows that at $A_{V}=3$ the effect brought by the non-linearity is $\sim 0.25 \mathrm{mag}$ in the Gaia $G_{\mathrm{BP}}-G_{\mathrm{RP}}$ colour for a solar type model. Therefore, a constant extinction coefficient (which could be derived by only computing the $\mathrm{BCs}$ with $A_{V}=0$ and $A_{V}=1 \mathrm{mag}$ ) for all $A_{V} \mathrm{~s}$ is not applicable. To consider the effect of extinction properly, we compute the BC tables with $A_{V}=[0,0.5,1,2,5,10,20]$ mag for each of the spectra in our database. These tables are used for interpolation in $A_{V}$ to derive BCs for any intermediate value of $A_{V}$.

In this section we show an example of isochrone fitting for NGC 2425 with Gaia DR2 data. The cluster is chosen

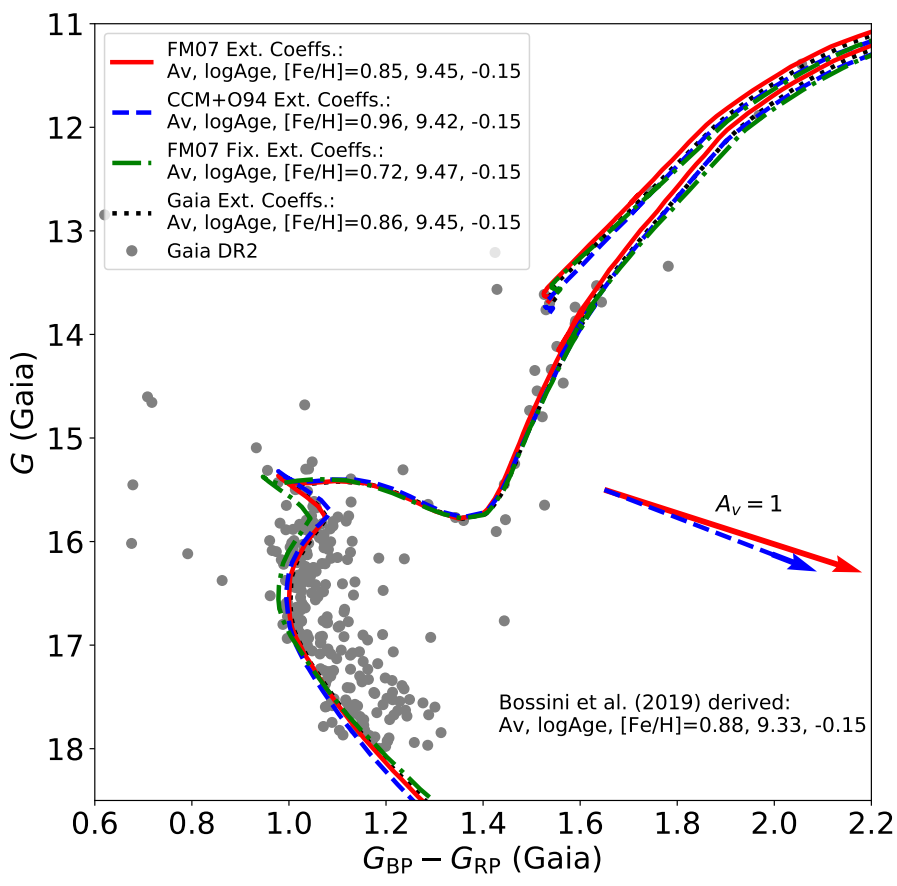

Fig. 5. Isochrone fitting for NGC 2425 with Gaia DR2 data. The PHOENIX and ATLAS9 models, and a smooth transition between the two are used for $\left(G_{\mathrm{BP}}-G_{\mathrm{RP}}\right)_{0} \gtrsim 0.94,\left(G_{\mathrm{BP}}-G_{\mathrm{RP}}\right)_{0} \lesssim 0.55$, and $0.94 \gtrsim$ $\left(G_{\mathrm{BP}}-G_{\mathrm{RP}}\right)_{0} \gtrsim 0.55$, respectively. For simplification, we fix the metallicity $([\mathrm{Fe} / \mathrm{H}]=-0.15 \mathrm{dex})$ and the true distance modulus (12.644 mag) as that derived from Bossini et al. (2019) for all the isochrones. The red solid line and blue dashed line are best-fit isochrones with variable extinction coefficients based on Fitzpatrick \& Massa (2007, FM07) and $\mathrm{CCM}+\mathrm{O} 94$ extinction laws, respectively. The vectors represent the corresponding fixed extinction coefficients. The green dot-dashed line is the best-fit isochrone with fixed extinction coefficients based on FM07 extinction law. The black dotted line shows the isochrone of the same age and metallicity as that of the red solid line, but with the extinction coefficients from that of Bossini et al. (2019).

because of its relatively large extinction and relatively old age. For this open cluster, Bossini et al. (2019) presented isochrone fitting parameters of $A_{V}=0.88 \mathrm{mag}$, a true distance modulus $\mathrm{DM}_{0}=12.644 \mathrm{mag}$, age $=2.15 \mathrm{Gyr}$, and $[\mathrm{Fe} / \mathrm{H}]=-0.15 \mathrm{dex}$. 

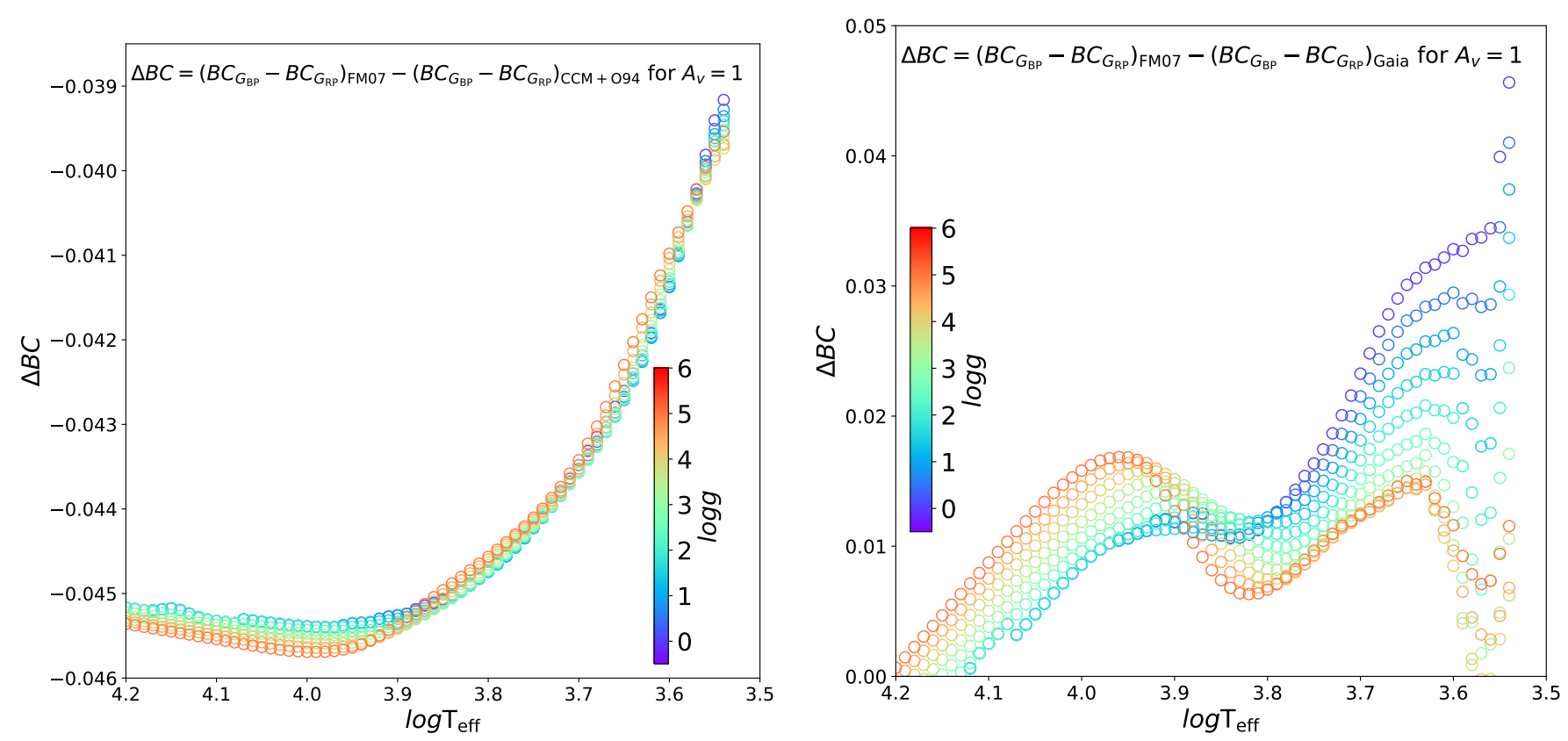

Fig. 6. Differences of $\mathrm{BC}_{G_{\mathrm{BP}}}-\mathrm{BC}_{G_{\mathrm{RP}}}$ for $A_{V}=1$ using different extinction laws as functions of $T_{\text {eff }}$ and $\log g$. Left: differences between using FM07 and CCM+O94 extinction laws. Right: differences between using our extinction coefficients based on FM07 extinction law and those of Bossini et al. (2019). The ATLAS9 models are used, but the PHOENIX models give consistent results.

Our intention is to demonstrate the effect of variable extinction coefficients, rather than obtain a perfect isochrone fitting. Therefore, we fix $[\mathrm{Fe} / \mathrm{H}]=-0.15$ dex and the true distance modulus (12.644 mag) as in Bossini et al. (2019), and vary only the way the extinction is applied to isochrones, and their ages. The best-fit isochrone with variable extinction coefficients based on the FM07 extinction law has an 0.19 Gyr older age and 0.11 mag smaller extinction than that with $\mathrm{CCM}+\mathrm{O} 94$ extinction law. The best-fit isochrones with fixed extinction coefficients based on the FM07 extinction law has an 0.13 Gyr older age and 0.13 mag smaller extinction than that with variable extinction coefficients. These numbers represent the uncertainties in isochrone fitting for an object with $A_{V} \sim 1 \mathrm{mag}$, when different extinction approaches are used. We also notice that our best-fit isochrone with FM07 extinction law predicts $\sim 0.67 \mathrm{Gyr}$ older age but similar extinction as that of Bossini et al. (2019), although we used the same PARSEC model set and atmosphere models. The extinction coefficients used in Bossini et al. (2019), which is from Gaia Collaboration (2018), are derived with FM07 extinction law. From this figure, we can see that the best-fit isochrone and the fitting parameters with variable extinction coefficients based on FM07 extinction law are very similar to that with extinction coefficients used in Bossini et al. (2019), thus the large difference in age should be due to other sources. However, this is out of the scope of this work.

To further investigate the effect of using different extinction law, we plot the BC differences with FM07, CCM+O94 and those of Bossini et al. (2019) in Fig. 6. In the range $\log T_{\text {eff }} \sim 3.5-3.8$, which comprises most of the NGC 2425 data, the colour difference between FM07 and CCM+O94 cases is about $0.41 \mathrm{mag}$, and this explains most of the differences in the derived physical parameters for isochrone fitting. The colour difference between FM07 and that of Bossini et al. (2019) is about $0.15 \mathrm{mag}$, to which some numerical errors might have contributed. Furthermore, at lower $\log T_{\text {eff }}$, there is a very large dispersion in the BCs owing to the variations in $\log g$. We thus find that, for the stellar atmospheres adopted in this work, the effect of using different extinction law and the gravity dependency at low temperatures is not small and should be included in extinction parameterisation.

The above discussions imply that to derive the stellar/cluster age and extinction more accurately, BCs that take into account the spectral type are urgently needed, especially for objects with large extinction.

\section{Summary and conclusions}

In this paper, we present a homogeneous database of BCs $\left(\mathrm{YBC}^{12}\right)$ for a large number of popular photometry systems, based on a variety of stellar spectral libraries we collected from the public domains or computed in our previous works. In this database, the $\mathrm{BC}$ tables both without extinction and with extinctions until $A_{V}=20$ are computed. Therefore, our YBC database provides a more realistic way to fit isochrones with a spectral type dependent extinction, and allow users to choose the atmosphere models that best for their science needs. The YBC database and software package are incorporated into the PARSEC isochrones provided via the web interface $\mathrm{CMD}^{13}$.

A potential application of this database is to incorporate it into large simulation programs, with the interpolation routine provided. For example, we implemented this database in TRILEGAL for stellar population simulations (Girardi et al. 2005; Girardi 2016).

Our database can be also useful for stellar evolution model comparison. For example, people studying star clusters may fit stellar evolution models from different groups to the observation data. However, different groups may provide photometric magnitudes with BCs from different libraries. Through our package, they can convert theoretical quantities into photometric magnitudes of the same libraries.

\footnotetext{
12 http://stev.oapd.inaf.it/YBC

13 http://stev. oapd.inaf.it/cgi-bin/cmd
} 
Y. Chen et al.: YBC bolometric corrections database

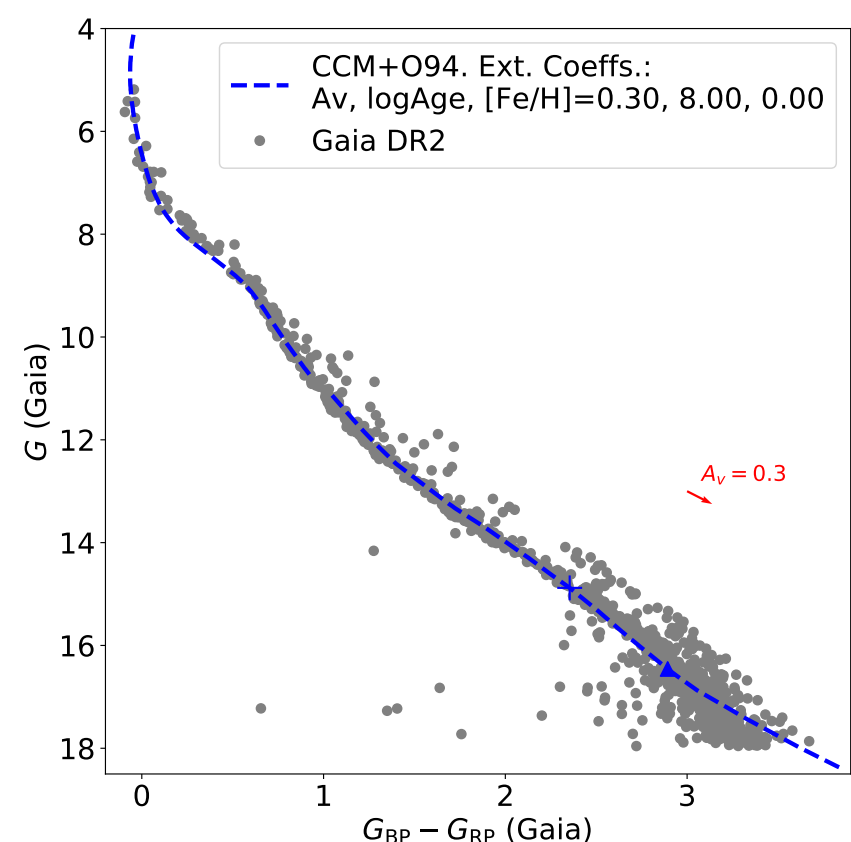

Fig. 7. Isochrone fitting for Melotte 22 with Gaia DR2 data. The atmosphere models are the same as those in Fig. 5. We fix the metallicity $([\mathrm{Fe} / \mathrm{H}]=0.0)$ and true distance modulus $(5.667 \mathrm{mag})$ as those derived from Bossini et al. (2019) for all the isochrones. The red cross and triangle represent stars of initial masses of 0.5 and $0.3 M_{\odot}$, respectively.

Finally, we also show an example of the isochrone fitting to a younger cluster, Melotte 22, with the Gaia DR2 data in Fig. 7. It presents a well-sampled main sequence, spanning almost 3.5 mag in $G_{\mathrm{BP}}-G_{\mathrm{RP}}$ colour. For this cluster, the extinction is very small, so that isochrones with both fixed and variable extinction coefficients do not differ much. It can be seen that isochrones adopting the present $\mathrm{BC}$ tables describe the entire sequence very well, except perhaps for the reddest and faintest stars, which can be affected by larger photometric errors, as well as by uncertainties in pre-main-sequence models and in their surface boundary conditions (see Chen et al. 2015).

Work is ongoing to compute $\alpha$-enhanced evolutionary models with and without rotation with the PARSEC code. Meanwhile, we are also computing $\alpha$-enhanced stellar atmosphere models with ATLAS12 code to extend the present database with $\alpha$-enhanced stellar spectral libraries. These spectral libraries will be incorporated into the web interface. We will also implement the MARCS atmosphere models (Gustafsson et al. 2008) into our database. The BCs derived from 3D atmosphere models will also be included ${ }^{14}$ in the future.

Acknowledgements. We acknowledge the support from the ERC Consolidator Grant funding scheme (project STARKEY, G.A. n. 615604). We thank Dr. Angela Bragaglia and Dr. Diego Bossini for useful discussions on clusters We thank Dr. Bengt Edvardsson for the help on MARCS atmosphere models. We thank Dr. Xiaodian Chen for kindly providing us the Python routine computing their extinction law. This research made use of Astropy, a communitydeveloped core Python package for Astronomy (Astropy Collaboration 2013 Price-Whelan et al. 2018). We are grateful to the referee for useful suggestions. Finally, we thank the editors for manuscript editing.

\section{References}

Allard, F., \& Hauschildt, P. H. 1995, ApJ, 445, 433

Allard, F., Hauschildt, P. H., Alexander, D. R., \& Starrfield, S. 1997, ARA\&A, 35,137

\footnotetext{
${ }^{14}$ http://www . astropy.org
}

Allard, F., Homeier, D., Freytag, B., \& Sharp, C. M. 2012, EAS Pub. Ser., 57, 3 Aringer, B., Girardi, L., Nowotny, W., Marigo, P., \& Lederer, M. T. 2009, A\&A, 503, 913

Aringer, B., Girardi, L., Nowotny, W., Marigo, P., \& Bressan, A. 2016, MNRAS, 457, 3611

Aringer, B., Marigo, P., Nowotny, W., et al. 2019, MNRAS, 487, 2133

Asplund, M., Grevesse, N., Sauval, A. J., \& Scott, P. 2009, ARA\&A, 47, 481

Astropy Collaboration (Robitaille, T. P., et al.) 2013, A\&A, 558, A33

Bessell, M. S. 1990, PASP, 102, 1181

Bessell, M. S., \& Brett, J. M. 1988, PASP, 100, 1134

Bessell, M. S., Castelli, F., \& Plez, B. 1998, A\&A, 333, 231

Bohlin, R. C., Gordon, K. D., \& Tremblay, P.-E. 2014, PASP, 126, 711

Bossini, D., Vallenari, A., Bragaglia, A., et al. 2019, A\&A, 623, A108

Bressan, A., Marigo, P., Girardi, L., et al. 2012, MNRAS, 427, 127

Caffau, E., Ludwig, H. G., \& Steffen, M. 2009a, Mem. Soc. Astron. It., 80, 643

Caffau, E., Maiorca, E., Bonifacio, P., et al. 2009b, A\&A, 498, 877

Caffau, E., Ludwig, H. G., Steffen, M., Freytag, B., \& Bonifacio, P. 2011, Sol. Phys., 268, 255

Cardelli, J. A., Clayton, G. C., \& Mathis, J. S. 1989, ApJ, 345, 245

Carnall, A. C. 2017, ArXiv e-prints [arXiv:1705.05165]

Casagrande, L., \& VandenBerg, D. A. 2014, MNRAS, 444, 392

Casagrande, L., \& VandenBerg, D. A. 2018a, MNRAS, 475, 5023

Casagrande, L., \& VandenBerg, D. A. 2018b, MNRAS, 479, L102

Casagrande, L., Ramírez, I., Meléndez, J., Bessell, M., \& Asplund, M. 2010. A\&A, 512, A54

Castelli, F., \& Kurucz, R. L. 2003, IAU Symp., 210, A20

Chen, Y.-P., Trager, S. C., Peletier, R. F., et al. 2014a, A\&A, 565, A117

Chen, Y., Girardi, L., Bressan, A., et al. 2014b, MNRAS, 444, 2525

Chen, Y., Bressan, A., Girardi, L., et al. 2015, MNRAS, 452, 1068

Chiavassa, A., Casagrande, L., Collet, R., et al. 2018, A\&A, 611, A11

Claret, A. 2000, A\&A, 363, 1081

Cohen, M., Wheaton, W. A., \& Megeath, S. T. 2003, AJ, 126, 1090

Evans, D. W., Riello, M., De Angeli, F., et al. 2018, A\&A, 616, A4

Fitzpatrick, E. L., \& Massa, D. 2007, ApJ, 663, 320

Fu, X., Bressan, A., Marigo, P., et al. 2018, MNRAS, 476, 496

Gaia Collaboration (Babusiaux, C., et al.) 2018, A\&A, 616, A10

Girardi, L. 2016, Astron. Nachr., 337, 871

Girardi, L., Bertelli, G., Bressan, A., et al. 2002, A\&A, 391, 195

Girardi, L., Groenewegen, M. A. T., Hatziminaoglou, E., \& da Costa, L. 2005, A\&A, 436, 895

Girardi, L., Dalcanton, J., Williams, B., et al. 2008, PASP, 120, 583

Girardi, L., Costa, G., Chen, Y., et al. 2019, MNRAS, 488, 696

Gräfener, G., Koesterke, L., \& Hamann, W.-R. 2002, A\&A, 387, 244

Grevesse, N., \& Sauval, A. J. 1998, Space Sci. Rev., 85, 161

Gustafsson, B., Edvardsson, B., Eriksson, K., et al. 2008, A\&A, 486, 951

Hamann, W.-R., \& Gräfener, G. 2004, A\&A, 427, 697

Ivezić, Ž., Kahn, S. M., Tyson, J. A., et al. 2019, ApJ, 873, 111

Jordi, C., Gebran, M., Carrasco, J. M., et al. 2010, A\&A, 523, A48

Koester, D. 2010, Mem. Soc. Astron. It., 81, 921

Koziol, Q., \& Robinson, D. 2018, HDF5, Computer Software, https://doi. org/10.11578/dc.20180330.1

Kurucz, R. L. 1979, ApJS, 40, 1

Kurucz, R. L. 2014, in Model Atmosphere Codes: ATLAS12 and ATLAS9, eds. E. Niemczura, B. Smalley, \& W. Pych, 39

Laidler, V., et al. 2005, Synphot Users Guide, Version 5.0 (Baltimore: STScI)

Luri, X., Brown, A. G. A., Sarro, L. M., et al. 2018, A\&A, 616, A9

Maíz Apellániz, J. 2006, AJ, 131, 1184

Maíz Apellániz, J., \& Weiler, M. 2018, A\&A, 619, A180

Mamajek, E. E., Torres, G., Prsa, A., et al. 2015, ArXiv e-prints [arXiv:1510.06262]

O’Donnell, J. E. 1994, ApJ, 422, 158

Oke, J. B. 1974, ApJS, 27, 21

Pauldrach, A., Puls, J., \& Kudritzki, R. P. 1986, A\&A, 164, 86

Pence, W. 1999, ASP Conf. Ser., 172, 487

Price-Whelan, A. M., Sipőcz, B. M., Günther, H. M., et al. 2018, AJ, 156, 123

Ramírez, I., \& Meléndez, J. 2005, ApJ, 626, 465

Rayner, J. T., Cushing, M. C., \& Vacca, W. D. 2009, ApJS, 185, 289

Sánchez-Blázquez, P., Peletier, R. F., Jiménez-Vicente, J., et al. 2006, MNRAS, 371,703

Sander, A., Hamann, W.-R., \& Todt, H. 2012, A\&A, 540, A144

Schmutz, W., Hamann, W.-R., \& Wessolowski, U. 1989, A\&A, 210, 236

Tange, O. 2011, https://doi .org/10.5281/zenodo. 16303

Taylor, M. 2017, Informatics, 4, 18

Thuan, T. X., \& Gunn, J. E. 1976, PASP, 88, 543

Todt, H., Sander, A., Hainich, R., et al. 2015, A\&A, 579, A75

Tremblay, P.-E., \& Bergeron, P. 2009, ApJ, 696, 1755

Wang, S., \& Chen, X. 2019, ApJ, 877, 116

Wells, D. C., Greisen, E. W., \& Harten, R. H. 1981, A\&AS, 44, 363 


\section{Appendix A: Usage of the web interface}

\section{Choose the filter sets}

2MASS JHKS
12 MASS JHKS
2MASS + Spitzer (IRAC+MIPS)
2MASS + Spitzer (IRAC+MIPS) + WISE
ti OGLE + 2MASS + Spitzer (IRAC+MIPS)
AKARI
BATC
2DENIS
DECAM (ABmags)
DMC 14 filters
DMC 15 filters
ESO/EIS (WFI UBVRIZ + SOFI JHK)
ESO/WFI
ESO/WFI2
CFHT/Megacam u*g'r''i''
HST/ACS HRC
HST/ACS WFC
HST/NICMOS AB
HST/NICMOS Vega
HST/STIS imaging mode, Vegamag
HST/WFPC2 (Vegamag, cf. Holtzman et al. 1995)

Fig. A.1. Filter selection step of the web interface, providing more than 70 filter combinations. It is updated with that of the CMD web interface.

\section{Choose the spectral libraries to use}

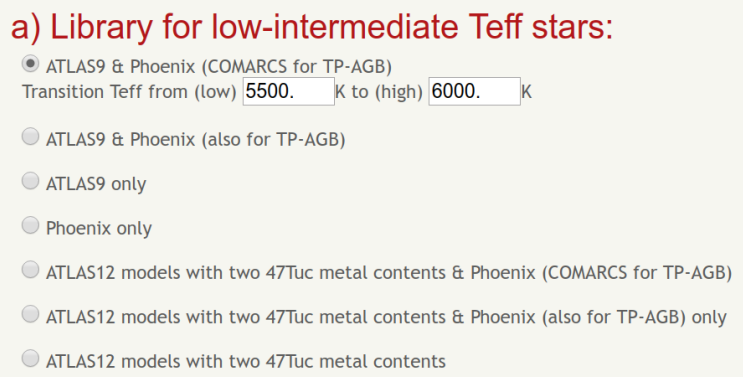

b) Library for high Teff stars:

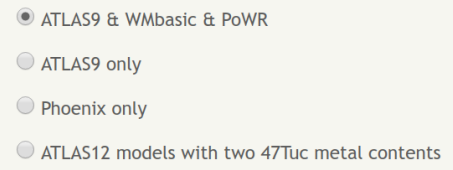

c) Library for AGB stars: - comarcs

Fig. A.2. Stellar spectral library selection step.

The YBC web page stores a user-submitted web form into an input parameter file, feeds this file as well as the user uploaded data file to the $\mathrm{C}$ executable for obtaining the $\mathrm{BCs}$, and saves the results into an ASCII file. The BCs are appended to the end of each line of a user-uploaded data file. Finally, the web page sends this result file to the browser for the user to download.

Figure A.1 shows the filter selection step of using the web interface. The list of filters come from that of the CMD web interface. It includes most of the popular filter sets and is frequently expanded.

Figure A.2 shows the library selection. In section (a), the user has different options for low-intermediate effective temperature stars. For those options with two libraries, a text element will show, which specifies the transition $T_{\text {eff }}$ between these two libraries. Section (b) is for hot stars and section (c) is for AGB stars.

In Sect. 3 of the web interface, as shown in Fig. A.3, the user can specify the interstellar extinction. For the moment, only the

\section{Interstellar extinction}

$A v=0 . \quad$ (Extinction curve: Cardelli et al. (1989) + O'Donnell (1994) with RV=3.1.)

The variation of extinction within the filter window, the effect of spectral type, and the non-lineararity as a function of $A v$ amount have been taking into account.

Circumstellar dust for RGB, RSG and TP-AGB are not included yet.

Fig. A.3. Extinction value setting step. More options will be added in the future.

\section{Stellar parameters of stars}

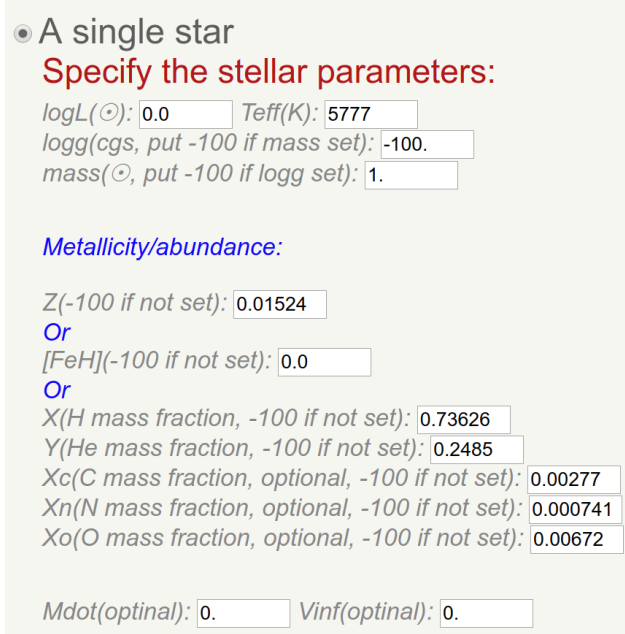

\section{A file containing stars}

Fig. A.4. Stellar parameter setting step for a single star.

\section{Stellar parameters of stars}

\section{A single star}

- A file containing stars

a) Upload your file: Choose File No file chosen

An example can be found at Here (with corresponding columns given below in default).

b) Specify indices of the columns (starting from 1) for the following parameters:

The number and order of the columns is not a matter. The first line is considered to be the header. Header and auxiliary information lines should start with '\#'.

$\log L(\odot): 5 \quad$ Teff(K): 6

$\log (\mathrm{cgs}$, put -100 if mass set): -100 .

mass $(\odot$, put -100 if logg set $): 8$

Metallicity/abundance:

$Z(-100$ if not set): -100

Or

$[\mathrm{Fe} / \mathrm{H}](-100$ if not set): -100 .

Or

$X(H$ mass fraction, -100 if not set $): 11$

$Y($ He mass fraction, -100 if not set $): 12$

$X_{C}(C$ mass fraction, -100 if not set): 13

$X n(N$ mass fraction, -100 if not set): 15

Xo(O mass fraction, -100 if not set $): 14$

Mdot(optinal): $10 \quad$ Vinf(optinal): -100.

Fig. A.5. Stellar parameter column specifying step for a user-uploaded star catalogue. 
Cardelli et al. (1989) extinction curve with the modification from O'Donnell (1994) is implemented, but we will soon add more options. Circumstellar dust for RSG and TP-AGB stars will also be considered in the next revision.

Then, the user can either specify the stellar parameters of a star (Fig. A.4) or upload the catalogue (Fig. A.5). In the case of uploading the catalogue, the user has to specify the column number of the stellar parameters. A 1 Gby maximum uploading limit is enforced, and ASCII format is supported. The surface chemical compositions are used to select the proper stellar spectral library for the stars. If the required chemical abundances are not provided by the user, a solar scaled abundance is used with the specified metallicity $Z$, which means all the relevant abundance ratios are the same as those in the Sun. Finally, a catalogue is generated for download by clicking on the submit button. 